\title{
Eine erfolgreiche Qualitätsinitiative: neues Zertifikat für Pharmaberater
}

\author{
Rainer Hofer ${ }^{\text {, }}$ \\ Peter Cavigelli ${ }^{b}$, \\ Christine Beyeler ${ }^{\text {, }}$ \\ Max Giger \\ a Institut für Medizinische \\ Lehre der Universität Bern \\ (IML) \\ b swiss health quality \\ association (shqa) \\ c Past-Präsident des Schweize- \\ rischen Instituts für ärztliche \\ Weiter- und Fortbildung \\ (SIWF)
}

* Zur besseren Lesbarkeit wird
für beide Geschlechter die männliche Form verwendet.

Korrespondenz:

Dr. phil. Rainer Hofer, Institut für Medizinische Lehre IML

Abt. Assessment und Evaluation Konsumstrasse 13

CH-3010 Bern

rainer.hofer@iml.unibe.ch

\section{Vorgeschichte}

Die Qualität der Beratung durch «Pharmaberater»* hängt von deren fachlichen und kommunikativen Kompetenzen ab. Sowohl Pharmaberater und Pharmaindustrie als auch Ärzte- und Apothekerschaft wünschten seit längerer Zeit einen Qualitätsnachweis. Die Schweizer Vertreter der Pharmaindustrie lancierten deshalb im Jahr 2005 das Projekt einer neuen schweizerischen Berufsprüfung für Pharmaberater. Diese sollten ab 2008 in einer freiwilligen Verbandsprüfung ihr Fachwissen dokumentieren und sich damit zertifizieren können. Die Prüfung soll das für eine kompetente, objektive Information und Beratung der Ärzte und Apotheker notwendige Fachwissen abbilden. Das Kompetenzniveau soll so angelegt werden, dass gutausgebildete und erfahrene Pharmaberater nach einer E-Learning- oder Präsenzweiterbildung die Prüfung bestehen. Ende 2010 soll die Mehrheit der in der Schweiz tätigen Pharmaberater zertifiziert sein. Das Projekt wurde durch die swiss health quality association (shqa) umgesetzt.

\section{Entwicklungsprozess}

Als Kompetenzpartner für Methodologie, Entwicklung, Durchführung und Evaluation der Prüfung wurde die Abteilung für Assessment und Evaluation (AAE) des Instituts für Medizinische Lehre (IML) der Universität Bern herangezogen. Die AAE/IML zeigte Bereitschaft, zusammen mit der shqa sowohl methodologisch wie auch technisch neue Wege zu beschreiten, um den Ansprüchen an eine effiziente und qualitativ hochstehende Berufsprüfung zu genügen. Die shqa stellte die Experten für den Lerninhalt und die AAE/IML diejenigen für die methodologische und informationstechnologische Entwicklung, Durchführung und Auswertung der Prüfung zur Verfügung.

\section{Plattform}

Für die Kommunikation zwischen den Experten entwickelte die AAE/IML zwei internetbasierte Applikationen: einerseits für die Entwicklung des Lernzielkatalogs und anderseits für die Erstellung von Fragen, deren Kontrollen und Übersetzungen. Vor allem das Erstellen, Überprüfen und Anpassen der Lernziele, inklusive deren Gewichtung und Festlegung der Taxonomie, wurde durch die Internetapplikation bedeutend erleichtert. Die weiteren Entwicklungen betrafen die Online-Prüfungsanmeldung sowie das Tool für die Prüfungsdurchführung via Internet.

\section{Une initiative qualité couronnée de succès: nouveau certificat pour conseillers pharmaceutiques}

En collaboration avec I'Institut de l'enseignement médical de l'université de Berne, les organisations suisses de l'industrie pharmaceutique ont développé pour les conseillers pharmaceutiques un examen basé sur les compétences et donnant lieu à un certificat. L'objectif est d'assurer la qualité du conseil dans les questions relatives aux médicaments. Les titulaires de ce certificat disposent de bonnes connaissances de la pharmacologie, des études spécifiques en la matière et du marché des médicaments. Ils comprennent la terminologie utilisée et peuvent informer le personnel médical de manière objective sur les médicaments spécifiques ou lui procurer les renseignements demandés. Cet examen a été développé sur une plate-forme basée sur l'Internet. Il est constamment accompagné d'un organe de surveillance composé de représentants de l'industrie, de l'enseignement médical et de la jurisprudence. Compté depuis sa création, le nombre de certificats délivrés s'est élevé à 605 en été 2010.

\section{Experten}

Für die Entwicklung des Lernzielkatalogs, für das Erstellen der Prüfungsfragen und für ihre Kontrollen waren drei voneinander unabhängige Expertengruppen zuständig. Während eine Gruppe die Verantwortung für den gesamten Lernzielkatalog innehatte, waren die Experten der beiden anderen Gruppen jeweils nur für einen spezifischen Lernzielbereich zuständig und hatten keine Einsicht in die anderen Bereiche.

\section{Lernzielkatalog}

Basierend auf dem Berufsbild «Pharmaberater 2010», das die Kompetenzanforderungen definierte, wurde im Jahr 2006 der Lernzielkatalog von 25 Experten aus der Pharmaindustrie, begleitet von einem ExpertenTeam der AAE/IML, entwickelt. Der Katalog umfasst 
Fachwissen zu Pharmakologie, Anatomie, Physiologie, Pathologie und klinische Studien (Gewicht: $75 \%$ ), sowie Grundlagen und Rahmenbedingungen des Arzneimittelmarktes in rechtlicher, betriebswirtschaftlicher und ethischer Hinsicht (Gewicht: 25\%). Als allgemeines Lernziel ist die Fähigkeit zu erwähnen, Informationsbedürfnisse von Medizinalpersonen $\mathrm{zu}$ verstehen, sowie ausgewogen und korrekt über Arzneimittel und damit zusammenhängende Fragen zu informieren.

\section{Blueprint}

Da die Gruppe «Lernzielkatalog» neben den Lernzielen auch ihre Gewichtung hinterlegt hatte, konnte daraus der Blueprint, das heisst das gewichtete Inhaltsverzeichnis der Prüfung abgeleitet werden. In den Prüfungen werden 70\% Wissens-, 24\% Verständnisund $6 \%$ Anwendungsfragen eingesetzt.

\section{Prüfungsform und Fragenentwicklung}

Da es sich in erster Linie um eine schriftliche Wissensprüfung handelt, wurde festgelegt, das Wissen, dessen Verständnis und Anwendung mit Wahlantwortfragen, sogenannten Multiple-Choice-Fragen, zu überprüfen.

25 Fachautoren aus der Pharmaindustrie erarbeiteten auf der Grundlage des Lernzielkatalogs und nach einer Schulung durch die AAE/IML die MultipleChoice-Fragen und erfassten diese in der internetbasierten Fragenverwaltung der AAE/IML. Diese Applikation erlaubt ein orts- und zeitunabhängiges Arbeiten.

Den Autoren wurden jeweils nur diejenigen Teile des Lernzielkatalogs vorgelegt, zu denen sie niveaugerechte Fragen $\mathrm{zu}$ formulieren hatten. In einer ersten Phase sollten 500 Fragen geschrieben, kontrolliert und übersetzt werden. Von dieser Zahl ausgehend, konnte - dank der Gewichtung - den Autoren mitgeteilt werden, wie viele Fragen in ihren Lernzielbereichen $\mathrm{zu}$ formulieren waren. Die Fragen wanderten

\section{Abbildung 1}

Der Fragenprozess.

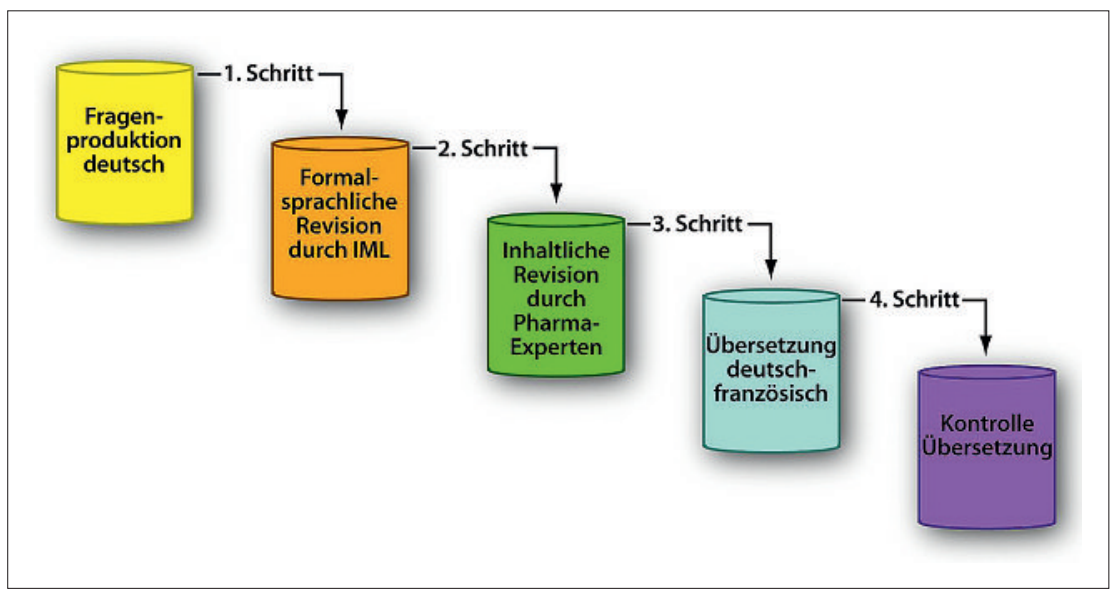

internetbasiert - auf ihrem «e-Tool-Weg» - von der Fragenproduktion zur formal-sprachlichen und danach zur inhaltlichen Revision, von dort zur Übersetzung und danach zur Kontrolle der Übersetzung, letztendlich in den Prüfungspool (Abb. 1). Kaum eine Frage hatte diesen Weg im ersten Anlauf geschafft. Oft wurden sie zur Überarbeitung wieder zum Autor zurückgeschickt und mussten danach die Kontrollen erneut über sich ergehen lassen. Vor allem bei der inhaltlichen Revision, in der die Fragen durch 30 weitere Experten aus der Pharmaindustrie nach denselben strengen Kriterien ebenfalls internetbasiert überprüft wurden, blieb manche Frage hängen. Mit diesem mehrstufigen Entwicklungsprozess wurde eine hohe Qualität der Fragen angestrebt.

\section{Prüfungsreglement und Prüfungskommission} Parallel zur Fragengenerierung wurde ein Prüfungsreglement erstellt, um aus juristischer Sicht das Regelwerk zur Prüfung zu definieren. Die Prüfungskommission umfasst Experten aus Ärzte- und Apothekerschaft, Pharmaberater und einen Juristen als Präsidenten. Sie hat die Aufgabe, sich über Prüfungsabläufe zu informieren, Prüfungsanalysen zur Kenntnis zu nehmen und daraus ableitend die definitiven Bestehensgrenzen zu bestimmen. Die Prüfungskommission kann auch Aufträge festlegen, die unter anderem zu einer Änderung des Prüfungsablaufs oder des Prüfungsreglements führen.

\section{Prüfungsanmeldung}

Die neu entwickelte Online-Prüfungsanmeldung der AAE/IML erlaubt den Pharmafirmen, die für die Zulassung notwendigen Daten der Kandidaten zu erfassen. Die Trägerorganisation der Prüfung, shqa, überprüft die Anmeldung und bestätigt die Zulassung elektronisch. Die AAE/IML, welche die Prüfungen an der Universität Bern durchführt, bestätigt schliesslich den Kandidaten die Teilnahme an dem von ihnen gewünschten Prüfungsdatum per E-Mail.

\section{Prüfungserstellung und Durchführung}

Jede Prüfung wird in der AAE/IML nach dem Zufallsprinzip zusammengestellt. Danach werden in der AAE/IML nur noch formale, aber keine inhaltlichen Kontrollen mehr durchgeführt. So wird gewährleistet, dass ein Prüfungsinhalt vor der Durchführung nicht bekannt wird. Die Kandidaten erhalten die Reihenfolge der Fragen nach dem Zufallsprinzip vorgelegt.

Die Prüfungslänge wurde zu Beginn auf 90 Fragen und die Prüfungszeit auf drei Stunden festgelegt. Die Prüfung wurde simultan von allen Kandidaten in einem Raum an Computern absolviert. Da an der Universität Bern keine grossen Räume mit Computern zur Verfügung stehen, wurde 2009 die Prüfungsdauer, unter Berücksichtigung der 2008 tatsächlich benötigten Beantwortungszeiten, auf 2 Stunden $30 \mathrm{Mi}$ nuten gekürzt. Die grossen Kandidatenzahlen in 2009 
veranlassten die Prüfungskommission, die Prüfungen ab 2010 mit Papier/Bleistift durchzuführen und die Prüfungsdauer wiederum auf drei Stunden zu verlängern.

Allen Kandidaten steht die Möglichkeit offen, eine Probeprüfung via Internet zu absolvieren, deren Frageninhalte die ganze Breite des Lernzielkatalogs abdeckt. Gleichzeitig konnten sich die Kandidaten mit dem Prüfungstool vertraut machen, das bis 2009 für die Prüfungen am Computer eingesetzt wurde.

\section{Ergebnisse der Prüfungsauswertung}

Bis Mitte 2010 wurden 9 zertifizierende Prüfungen durchgeführt. Tabelle 1 zeigt über alle Prüfungen die Anzahl der Teilnehmenden, die mittlere Leistung (korrekt beantwortete Fragen), die Anzahl der erfolgreichen Absolventen (Erfolgsquote) sowie die mittlere Messzuverlässigkeit (Reliabilität). 230 Kandidaten $(31,3 \%)$ haben eine französische Version der Prüfung bearbeitet, 280 (38,0\%) waren Männer.

Gemäss Prüfungsreglement sind $2 / 3$ aller Aufgaben richtig zu beantworten. Zusätzlich muss pro Lernzielgebiet mindestens die Hälfte der Fragen korrekt beantwortet werden. Unter Berücksichtigung der Prüfungsschwierigkeit sowie der Häufigkeitsverteilung legt die Prüfungskommission jeweils die definitive Bestehensgrenze je Prüfung fest. Bis Mitte 2010 haben 605 Pharmaberater die Prüfung bestanden und damit das Zertifikat erworben.

\section{Diskussion und Ausblick}

Es war von Beginn an das Ziel, die Qualität der Prüfung systematisch zu erfassen und zu beurteilen sowie Verbesserungen laufend einfliessen zu lassen. So enthält jeder Prozess das Element der ständigen Evaluation. Bereits beim Lernzielkatalog wurden Feedback-Schlaufen eingebaut, um die richtige «Flughöhe» des Wissensniveaus zu garantieren. Die Auswertung der Prüfungen und Hinweise von Kandidaten werden systematisch evaluiertund einem jährlichen Überprüfungs-und Anpassungsprozess unterzogen. Durch die jährliche Überprüfung und allfällige Anpassung der Gewichtung der Prüfungsinhalte und der Lernziele lässt sich das Niveau der Prüfung nach Bedarf mittelfristig regulieren. So können Erfahrungen aus dem Prüfungsprozess oder den veränderten Anforderungen des Umfelds systematisch in die Aus-/Weiterbildung und Prüfungsvorbereitung einfliessen.

Die Messzuverlässigkeiten (Reliabilität, Cronbachalpha) der Prüfungen betrugen bei einer Ausnahme immer mehr als 0,8 (Bereich von 0,77 bis 0,95 ) und entsprachen somit internationalen Standards, die für Prüfungen mit einschneidenden Konsequenzen für die Kandidaten eingehalten werden müssen, obwohl Eingriffe in Prüfungsdauer und Prüfungsmedium vorgenommen wurden. Somit genügen die gewählte Länge von 90 Fragen und die Dauer von drei bzw. zweieinhalb Stunden zur summativen Beurteilung der Kandidaten.

Wird davon ausgegangen, dass in der Schweiz aktuell circa 1000 Pharmaberater tätig sind, kann geschlossen werden, dass bis heute mehr als $60 \%$ dieser Berufsgruppe zertifiziert sind. Eine Auswirkung dieser Zertifizierung auf die Beratung der Ärzte und Apotheker wurde noch nicht evaluiert.

Die Verbandsprüfung «zertifizierte/r Pharmaberater/in» wurde in enger Zusammenarbeit zwischen Wissenschaft und Wirtschaft entwickelt. Dabei wurde in verschiedener Hinsicht Neuland betreten, und praxisrelevante Innovationen wurden geschaffen. Das forschungsbasierte Wissen aus der Universität wird mit dieser Prüfung in der Wirtschaft zielgerichtet eingesetzt: eine innovative Methodologie, der Einsatz internetbasierter Technologie und Prozesse sowie ein systematisches Qualitätsmanagement. Die mit grossem Aufwand erarbeitete Prüfung dürfte für ähnlich gelagerte Projekte im In- und Ausland eine Vorreiterrolle bilden.

\section{Dank}

Allen Mitarbeitern der Ärzte- und Apothekerschaft und der Pharmaindustrie, die beim Erarbeiten des Lernzielkataloges, der Fragen oder der Reglemente mitwirkten, sei hiermit herzlich gedankt. Die Autoren danken den Mitgliedern der Prüfungskommission für die wertvollen Hinweise zum Artikel.

\begin{tabular}{|c|c|}
\hline Prüfungscharakteristika von März 20 & Juni 2010. \\
\hline Total & \\
\hline Anzahl Kandidaten & 736 \\
\hline Durchgeführte Prüfungen & 9 \\
\hline $\begin{array}{l}\text { Korrekt beantwortete Fragen } \\
\text { (Mittelwert } \pm \text { Standardabweichung) }\end{array}$ & $70,7 \% \pm 10,9 \%$ \\
\hline $\begin{array}{l}\text { Anzahl erfolgreiche Absolventen } \\
\text { (Erfolgsquote) }\end{array}$ & $605(82,2 \%)$ \\
\hline $\begin{array}{l}\text { Reliabilität (Cronbach- } \alpha \text { ) } \\
\text { (Mittelwert } \pm \text { Standardabweichung) }\end{array}$ & $0,84 \pm 0,05$ \\
\hline
\end{tabular}

